\title{
Propostas didáticas de experimentação: uma análise prévia da Revista do Ensino do Rio Grande do Sul (1951-1959)
}

\author{
Propuestas didácticas de experimentación: un análisis previo de la Revista \\ de la Enseñanza de Rio Grande do Sul
}

\author{
Didactic proposals for experimentation: a previous analysis of Journal of \\ Teaching of Rio Grande do Sul
}

Vitor Garcia Stoll ${ }^{1}$

Alessandro Carvalho Bica ${ }^{2}$

Cadidja Coutinho ${ }^{3}$

\begin{abstract}
Resumo
Este trabalho apresenta um recorte dos estudos desenvolvidos no Mestrado em Ensino da Universidade Federal do Pampa (UNIPAMPA) - Campus Bagé. Objetivou-se realizar um levantamento sobre as propostas didáticas de experimentações contidas nas Seções "Observando" e "Observações e Experiências" da Revista do Ensino do Rio Grande do Sul, no período de 1951 a 1959, e os conteúdos científicos abordados pelas mesmas. Caracterizase como uma pesquisa qualitativa, sendo a abordagem técnico-metodológica pautada em análise documental, dados numéricos e estatística descritiva. Os dados empíricos foram obtidos a partir dos acervos disponíveis no Repositório Digital Tatu, vinculado a UNIPAMPA. E o corte temporal demarca a década inicial da segunda fase da revista. No período delimitado foram encontrados 34 exemplares da Revista do Ensino/RS no Repositório. Os anos com mais edições disponíveis foram 1957 e 1959, representando 22\% e 19\% da amostragem, respectivamente. E os de menor representação, 1951 e 1956, com uma edição cada (3\%). Constatou-se que 15 edições (50\%) apresentavam experimentações com predominância disciplinar em Biologia, 08 em Física (27\%) e 05 em Química (17\%). Além disso, duas edições $(6 \%)$ possuíam tanto experimentações de Física quanto de Química. Concluiu-se que tal material, que na época servia como suporte técnico-pedagógico para os professores, assemelha-se às práticas experimentais utilizadas atualmente nas aulas Ciências da Natureza.
\end{abstract}

Palavras-Chave: Análise documental; Década de 1950; Ensino de Ciências da Natureza; Experimentação; Revista do Ensino/RS.

\section{Resumen}

Este trabajo presenta un recorte de los estudios desarrollados en el Máster en Enseñanza de la Universidad Federal de Pampa (UNIPAMPA) - Campus Bagé. Se pretendió realizar un levantamiento sobre las propuestas didácticas de ensayos contenidas en las Secciones "Observando" y "Observaciones y Experiencias" de la Revista de la Enseñanza de Rio Grande do Sul, en el período de 1951 a 1959, y los contenidos científicos abordados por las mismas. Se caracteriza como una investigación cualitativa, siendo el abordaje técnico-metodológico pautado en análisis documental, datos numéricos y estadística descriptiva. Los datos empíricos fueron obtenidos a partir de los acervos disponibles en el Repositorio Digital Tatu, vinculado a UNIPAMPA. Y el corte temporal demarca la década inicial de la segunda fase de la revista. En el período delimitado fueron encontrados 34 ejemplares de la Revista de la Enseñanza / RS en el Repositorio. Los años con más ediciones disponibles fueron 1957 y 1959,

\footnotetext{
${ }^{1}$ Licenciado em Ciências da Natureza; Universidade Federal do Pampa - UNIPAMPA; Bagé, Rio Grande do Sul, Brasil; vitorgarciastoll@gmail.com

${ }^{2}$ Doutor em Educação; Universidade Federal do Pampa - UNIPAMPA; Bagé, Rio Grande do Sul, Brasil; alessandrobica@gmail.com

${ }^{3}$ Doutora em Educação em Ciências: Química da Vida e Saúde; Universidade Federal do Pampa - UNIPAMPA; Dom Pedrito, Rio Grande do Sul, Brasil; cadidjabio@gmail.com
} 
representando el 22\% y el 19\% del muestreo, respectivamente. Y los de menor representación, 1951 y 1956 , con una edición cada uno (3\%). Se constató que 15 ediciones $(50 \%)$ presentaban ensayos con predominio disciplinario en Biología, 08 en Física (27\%) y 05 en Química (17\%). Además, dos ediciones (6\%) poseían tanto experimentaciones de Física y de Química. Se concluyó que tal material, que en la época servía como soporte técnico-pedagógico para los profesores, se asemeja a las prácticas experimentales utilizadas actualmente en las clases de Ciencias de la Naturaleza.

Palabras claves: Análisis documental; Década de 1950; Enseñanza de Ciencias de la Naturaleza;

Experimentación; Revista de la Enseñanza/RS.

\begin{abstract}
This paper presents a review of the studies developed in the Masters in Teaching of the Federal University of Pampa (UNIPAMPA) - Campus Bagé. The aim of this study was to make a survey about the didactic proposals of experiments contained in the "Observing" and "Observations and Experiences" sections of the Revista do Ensino do Rio Grande do Sul, from 1951 to 1959, and the scientific content addressed by them. It is characterized as a qualitative research, being the technical-methodological approach based on documentary analysis, numerical data and descriptive statistics. The empirical data were obtained from the collections available in the Digital Repository Tatu, linked to UNIPAMPA. And the temporal cut marks the initial decade of the second phase of the magazine. In the delimited period were found 34 copies of the Journal of Teaching / RS in the Repository. The years with the most editions available were 1957 and 1959, representing $22 \%$ and $19 \%$ of the sampling, respectively. And those of smaller representation, 1951 and 1956, with one edition each (3\%). It was verified that 15 editions (50\%) presented experiments with a predominance of disciplines in Biology, 08 in Physics (27\%) and 05 in Chemistry (17\%). In addition, two editions (6\%) had both physics and chemistry experiments. It was concluded that such material, which at the time served as technical and pedagogical support for teachers, is similar to the experimental practices currently used in the Nature Science classes.
\end{abstract}

Keywords: Documentary analysis; Experimentation; Journal of Teaching/RS; Teaching of Natural Sciences; 1950 s.

\title{
1. Introdução
}

A Revista do Ensino do Rio Grande do Sul (RE/RS) foi um periódico de grande relevância na história da educação brasileira em sua época de distribuição, considerada um dos meios mais importantes de disseminação de discursos sobre educação e ensino. Apresentava em seu conteúdo um vasto material com sugestões de práticas para serem trabalhadas em sala de aula, servindo como guia e suporte para muitos professores do magistério (PEREIRA, 2010; DUARTE, 2009).

Bastos (1997) classifica a referida revista como uma publicação de imprensa educacional e de ensino, cujo objetivo principal é guiar e auxiliar os professores nas suas práticas em sala de aula. Como imprensa pedagógica a autora caracteriza os jornais, boletins, revistas e magazines desenvolvidos por professores, pelo Estado ou por outras instituições (sindicatos, associações e partidos políticos) tendo como público-alvo professores e estudantes.

De acordo com Bastos (1997 apud Pereira, 2010) “[...] a imprensa periódica pedagógica é um instrumento de pesquisa que se apresenta como importante fonte de 
informação para a história da educação, que deve, enquanto tal, submeter-se ao crivo de uma adequada crítica documental". Sendo assim, a análise desse tipo de documento, como proposto pelo presente trabalho, possibilita a multiplicidade de perspectivas para a compreensão da história da educação e do ensino, permite avaliar as políticas organizacionais, as preocupações sociais, ideológicas e práticas educativas da época.

Criada na década de 1930, a RE/RS teve sua primeira fase de distribuição de 1939 a 1942, com 26 exemplares produzidos. Após nove anos de interrupção, a partir das iniciativas das professoras primárias Maria de Lourdes Gastal, Gilda Garcia Bastos e Abigail Teixeira a revista voltou a circular em 1951, finalizando a segunda fase de edição em 1978, período que foram produzidas 170 edições, tendo tiragem inicial de 5.000 exemplares, chegou a atingir a marca de 55.000 na década de 1970 (FARIA, 2017; PEREIRA, 2010; DUARTE 2009).

Pereira (2010) destaca que eram publicados em média oito a dez números por ano, contendo cerca de 80 páginas cada. A circulação dava-se pelo sistema de assinaturas e sua abrangência era nacional e internacional, transpondo em grande escala os limites geográficos do Estado do Rio Grande do Sul. Como exemplo, o autor apresenta um recorte do discurso da professora Maria de Lourdes Gastal, fundadora e diretora da revista por 15 anos. Em sua fala, pronunciada no jantar de comemoração dos 26 anos do periódico, a professora salienta a importância da RE/RS para os docentes do primário e conta a sua rápida expansão, sendo distribuída para países como Argentina, Chile, Uruguai, Portugal, Espanha, Itália e Alemanha. Além disso, também cita as cartas recebidas de grandes mestres como Anísio Teixeira, Amaral Fontoura e Juracy Silveira, no qual, expressam entusiasmo e fé na missão da Revista do Ensino.

Faria (2017, p. 186) enfatiza que a segunda fase "[...] tinha como objetivo oferecer orientações didáticas que pudessem ajudar os professores primários na prática diária de suas atividades" através da divulgação de diretrizes técnico-pedagógicas, materiais didáticos e legislação relativa ao ensino. E complementa através de Bastos (2004) que nessa fase:

\footnotetext{
A Revista do Ensino volta-se a dar orientação didático-pedagógica aos professores do ensino primário e pré-primário, através de sugestões de recursos de ensino. Além disso, procura auxiliar o professor no dia-a-dia da sala de aula, [...] fomentando subsídios de ensino de aplicação imediata em sala de aula e dirigindo o cotidiano da escola primária (BASTOS, 2004, p. 4-5 apud FARIA, 2017, p. 186).
}

Desse modo, percebe-se que a revista serviu como base e referência pedagógica, didática e metodológica em sua época de circulação. Por ter como foco os professores do ensino primário, apresentava em seu conteúdo orientações específicas e recursos visuais para 
serem utilizados em sala de aula, bem como tratava de temas específicos como música, meio ambiente, datas comemorativas, entre outros (PEREIRA, 2010; SARDAGNA, 2008).

Sardagna (2008) destaca que a estrutura da RE/RS e as seções nelas contidas modificaram-se ao longo dos anos, possivelmente em razão das trocas de editoras responsáveis pelas publicações, o que demarca a necessidade de recorte temporal para o desenvolvimento dessa pesquisa. Optou-se por analisar as edições disponíveis entre o período de 1951 a 1959, demarcando a década inicial da segunda fase de distribuição.

No que tange ao Ensino de Ciências, a revista possui poucas seções específicas destinadas a essa área do conhecimento. Neste artigo, buscou-se realizar um levantamento sobre as propostas didáticas de experimentações contidas nas Seções "Observando" e "Observações e Experiências" da RE/RS no período delimitado, e os conteúdos científicos abordados pelas mesmas.

Com base nesses pressupostos e reconhecendo a relevância histórica dessa revista, essa pesquisa une duas áreas distintas: História da Educação e Ensino de Ciências. Possibilita não só um resgate histórico, mas também evidencia traços de práticas que podem ser utilizadas no contexto educacional atual.

\section{Caminhos da pesquisa}

Metodologicamente essa pesquisa possui natureza qualitativa, alicerçada em aspectos da realidade que não podem ser quantificados, ou seja, que não se restringem à operacionalização de variáveis (MINAYO, 2002). De acordo com Gil (2002, p. 133), esse tipo de investigação define-se como "[...] uma sequência de atividades, que envolve a redução dos dados, a categorização desses dados, sua interpretação e a redação do relatório", podendo variar de acordo com a extensão da amostra, instrumentos de pesquisa e pressupostos teóricos.

Quanto aos procedimentos técnicos caracteriza-se como um levantamento documental, pois, o objeto de estudo são as edições da RE/RS. Logo, a abordagem técnico-metodológica pauta-se na análise documental, que consiste "[...] no conjunto de procedimentos efetuados com o fim de expressar o conteúdo de documentos, sob formas destinadas a facilitar a recuperação de informações" (CUNHA, 1989, p. 40). Assim, os principais momentos da análise documental são analisar, sintetizar e representar, podendo utilizar-se também de recursos como classificação, indexação e elaboração de resumos (BICA, 2013). 
Conforme corrobora Bica (2013), presume-se que o objetivo primário da perspectiva da análise documental seja representar para recuperar. Por esse motivo, optou-se por apresentar os resultados através de dados numéricos e estatísticas descritivas, distribuídas em quadros e tabelas que possibilitam ao leitor identificar a síntese das informações analisadas pelos pesquisadores.

Os dados empíricos foram obtidos a partir dos acervos disponíveis no Repositório Digital Tatu, vinculado a Universidade Federal do Pampa - UNIPAMPA. Este repositório nasceu das iniciativas do projeto “As Políticas Públicas de Formação de Professores em impressos pedagógicos: O caso da Revista do Ensino do Rio Grande do Sul (1951-1978)”, no qual, desde 2015 tem realizado ações de digitalização e disponibilização de algumas edições da referida revista (UNIPAMPA, 2019).

Atualmente, integrado ao projeto "Educação, História e Políticas na região de abrangência da Universidade Federal do Pampa" e ao Grupo de Estudos em Educação, História e Narrativas (GEEHN), o repositório tem por objetivo inventariar fontes de pesquisa relacionadas à história da educação, compondo um acervo digital acessível para outros pesquisadores (UNIPAMPA, 2019). Em 2018 o Tatu foi ampliado, contando atualmente com centenas de obras, que incluem livros, cartilhas, revistas e produções acadêmicas, que além de resgatar à história da educação, podem servir de suporte aos pesquisadores de diversas áreas do conhecimento.

Nesta pesquisa, analisaram-se as edições disponíveis da RE/RS entre o período de 1951 a 1959. Inicialmente realizaram-se a leitura flutuante das edições, procurando indícios que demarquem o Ensino de Ciências. Nesse primeiro contato com o acervo, encontram-se as Seções "Observando" e "Observações e Experiências", foco deste estudo, o qual dispõe de propostas didáticas de experimentações para serem desenvolvidas em sala de aula.

Evidenciou-se que a seção "Observando" foi publicada até meados de 1953, a partir da edição de março o nome foi editado para "Observações e Experiências", porém continuou com o mesmo objetivo. Sendo assim, na apresentação dos resultados não haverá distinção entre as seções. As experimentações foram categorizadas por componente curricular, de modo que, possam expressar os conteúdos científicos abordados pelas mesmas.

\section{Resultados e Discussões}


No período delimitado (1951-1959), foram encontrados 34 exemplares da RE/RS no Repositório Tatu. O Quadro 1 apresenta as edições mensais disponíveis para análise e também aquelas em que não foram encontradas as Seções "Observando" e "Observações e Experiências". No qual, percebeu-se que os anos com mais edições disponíveis foram 1957 e 1959, representando $22 \%$ e $19 \%$ da amostragem, respectivamente. $\mathrm{E}$ os de menor representação foram 1951 e 1956, com uma edição cada (3\%).

\begin{tabular}{|c|c|c|c|c|c|c|c|c|c|c|c|c|c|}
\hline \multirow[b]{2}{*}{$\begin{array}{c}\text { Ano de } \\
\text { Publicação }\end{array}$} & \multicolumn{13}{|c|}{ Edições mensais disponíveis para análise } \\
\hline & $\underset{\mathbf{\Xi}}{\stackrel{0}{二}}$ & $\overrightarrow{0}$ & $\stackrel{\Xi}{\Sigma}$ & 节 & $\stackrel{\circ}{\stackrel{0}{3}}$ & $\Xi$ & $\Xi$ & $\stackrel{0}{0}$ & $\ddot{\omega}$ & 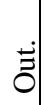 & $\begin{array}{l}z \\
z\end{array}$ & فे & $\stackrel{\tilde{\pi}}{0}$ \\
\hline 1951 & & & & & & & & & & & $\mathbf{x}$ & & 01 \\
\hline 1952 & & & $\mathbf{X}$ & $\mathbf{x}$ & $\mathbf{X}$ & & & $\mathbf{x}$ & & & & & 04 \\
\hline 1953 & & & $\mathbf{X}$ & & & & & & $\mathbf{x}$ & & & & $\mathbf{0 2}$ \\
\hline 1954 & & & $\mathbf{X}$ & $\mathbf{x}$ & $\mathbf{X}$ & $\mathbf{x}$ & & & & $\mathbf{x}$ & & & 05 \\
\hline 1955 & & & $\mathbf{X}$ & & & $\mathbf{x}$ & & $\mathbf{x}$ & & & & & $\mathbf{0 3}$ \\
\hline 1956 & & & & & & & & & & $\mathbf{x}$ & & & 01 \\
\hline 1957 & & & $\mathbf{X}$ & & $\mathbf{x}$ & & & $\mathbf{x}$ & $\mathbf{x}$ & $\mathbf{x}$ & $\mathbf{x}$ & & 06 \\
\hline 1958 & & & $\mathbf{X}$ & & $\mathbf{x}$ & & & & $\mathbf{x}$ & $\mathbf{x}$ & $\mathbf{x}$ & & 05 \\
\hline 1959 & & & $\mathbf{X}$ & $\mathbf{x}$ & & $\mathbf{x}$ & & $\mathbf{x}$ & $\mathbf{x}$ & $\mathbf{x}$ & $\mathbf{x}$ & & 07 \\
\hline
\end{tabular}

$\mathbf{x}$ - Edições analisadas;

- Não foram encontradas as Seções Observações ou Observações e Experiências;

- Anos com menos edições disponíveis;

- Anos com mais edições disponíveis

Quadro 1 - Edições da Revista do Ensino do Rio Grande do Sul analisadas.

Fonte: Autores (2019).

$\mathrm{O}$ arquivo mais antigo da RE/RS disponível no Repositório Tatu é datado de novembro de 1951 e apresenta a terceira publicação da revista na segunda fase de distribuição (Ano I - n. 3). Já a edição de outubro de 1956 demarca o período em que a revista passou a ser propriedade privada de uma professora. De acordo com Duarte (2009, p. 38) “[...] de maio a novembro de 1956, o periódico passou a ser propriedade privada da professora Maria de Lourdes Gastal" e "[...] somente em 11 de dezembro de 1956, a revista passou a ser uma publicação oficial sob a supervisão técnica do CPOE/RS - Centro de Pesquisas e Orientação Educacional do Rio Grande do Sul”.

Em apenas quatro exemplares (12,5\%) não foram encontrados as seções analisadas, são eles: maio de 1958 e março, outubro e novembro de 1959. Salienta-se que essas edições disponíveis no Repositório Tatu estavam com páginas incompletas. Ao analisar o sumário verificou-se a presença dessas seções, o que indica que a experimentação é um elemento presente e contínuo nas publicações da época. 
De acordo com Giordan (1999) a experimentação, além de despertar interesse nos estudantes, também contribui para um caráter motivador, lúdico e vinculado aos sentidos. Se utilizada de maneira contextualizada, vinculada às aulas teóricas e as discussões em grupo, a experimentação pode contribuir significativamente no processo de ensino e aprendizagem de Ciências e na discussão dos fenômenos físicos (ROSITO, 2008).

Além dessas contribuições, Oliveira (2010) destaca que a experimentação pode proporcionar a (re)construção de conceitos científicos com a finalidade de relacionar Ciência, Tecnologia e Sociedade ao cotidiano dos estudantes. Igualmente, melhora a relação entre professor-estudante, aguça a curiosidade, estimula o trabalho em grupo e aprimora a capacidade criativa e a iniciativa pessoal. (ROSITO, 2008).

Contudo, percebeu-se que as experimentações contidas na RE/RS estão fundamentadas no empirismo, possivelmente, devido ao contexto histórico da década de 1950. De acordo com Lima (2016, p. 27) “o empirismo está pautado na ideia de que o conhecimento é proveniente da experiência do indivíduo com o meio físico, levando em consideração a força dos sentidos, [...] sendo o sujeito um elemento passivo e o meio determinante para construção do conhecimento". Ou seja, nessa perspectiva epistemológica o conhecimento vem de fora para dentro, gerado a partir do objeto e não do sujeito.

No que compete a experimentação, Lima (2016) apresenta através de Destch (1997) que para ser considerada de cunho empirista precisa ter características específicas, das quais se destacam algumas: (1) desenvolver o experimento a partir de observações e informações precisas; (2) fazer registros sistemáticos; (3) ter o princípio da replicabilidade; (4) possibilidade de formular hipóteses a partir do acúmulo de informações, dentre outras. A Figura 1 apresenta uma das propostas de experimentações contida na edição de novembro de 1951 da RE/RS, no qual, propõe que os estudantes tragam amostras de solos de diferentes locais, plantem sementes iguais em cada vaso e observem qual amostra é melhor para o desenvolvimento das plantas.

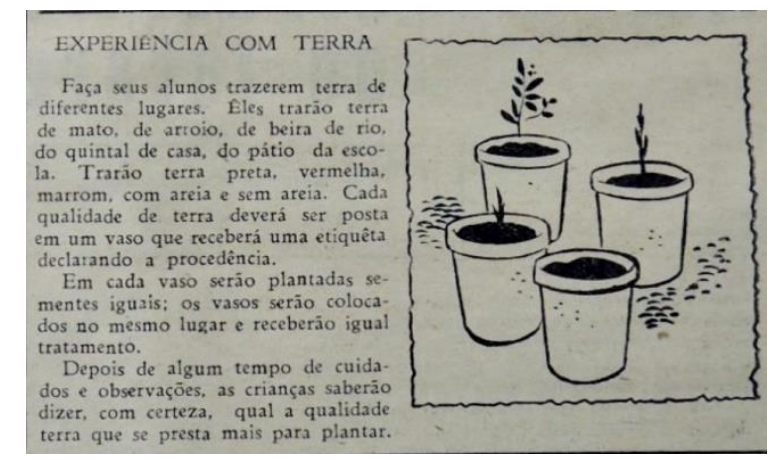

Figura 1 - Experimentação com Terra contida na edição de novembro de 1951 da RE/RS. 
Fonte: Revista do Ensino do Rio Grande do Sul (novembro de 1951, p. 17).

Já a Figura 2 apresenta um fragmento da proposta de experimentação contida na edição de março de 1955, que traz diversos experimentos com a temática da água. No primeiro, orienta os estudantes a recolherem amostras de água de distintos locais e anotar e comparar as diferenças entre elas, tais como cheiro e sabor. Enquanto que, no segundo propõe observar os diferentes estados da matéria.

1) Recolher água de poço, de rio, de mar, de chuva, etc. Compará-las e anotar as diferenças que notar, quanto à transferència, cheiro e sabor.

2) Fazer experiéncias para observar as diferentes estados da água: a) derreter gelo; b) ferver água; c) chegar um prato ao vapor dágua para observá-lo liquéfazer-se.

Figura 2 - Experimentação sobre água contida na edição de março de 1955 da RE/RS.

Fonte: Revista do Ensino do Rio Grande do Sul (março de 1955, p. 20).

Constatou-se que em ambas as experimentações o foco é a observação e a comparação, demarcando a experimentação empírica. Tal concepção adotada desde a década de 1950 ainda é utilizada por alguns professores atualmente. Carvalho e colaboradores (2000) enfatizam que é o professor que propõe a experimentação, logo tem autonomia para criar problemas a ser resolvido, gerar ideias e conduzir discussões, bem como promover oportunidades de reflexão e trabalho coletivo. Desse modo, acredita-se que as experimentações contidas na RE/RS podem ser utilizadas de forma construtivista, a partir de adaptações e da introdução de questões problemas.

Com base nesses pressupostos e reconhecendo a relevância da experimentação para com o Ensino de Ciências, a segunda etapa dessa pesquisa consistiu em categorizar as experimentações contidas na seção supracitada por predominância disciplinar, conforme Figura 3.

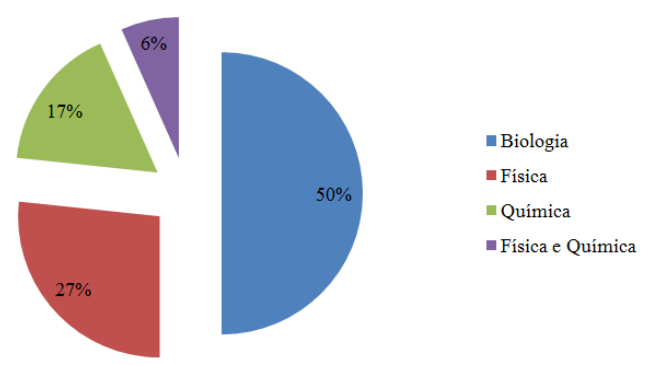


Figura 3 - Predominância disciplinar das experimentações da RE/RS.

Fonte: Autores (2019).

Identificou-se que 15 edições (50\%) apresentavam experimentações com predominância disciplinar em Biologia, oito em Física (27\%) e cinco em Química (17\%). Além disso, as edições de maio de 1952 e junho de 1959 possuíam tanto experimentações de Física (alavancas e ilusão de ótica) quanto de Química (densidade e combustão). Para exemplificar as possibilidades da utilização das experimentações analisadas, a Tabela 1 mostra os possíveis conteúdos científicos que podem ser desenvolvidos em sala de aula.

Tabela 1 - Possíveis conteúdos científicos abordados nas experimentações analisadas

\begin{tabular}{|c|c|c|c|c|c|}
\hline Ano & Mês & Título da Seção & Conteúdo Científico & Pag. & $\begin{array}{c}\text { Predominância } \\
\text { disciplinar }\end{array}$ \\
\hline 1951 & Nov. & Observando plantas & Solo & 17 & Biologia \\
\hline 1952 & Mar. & Termômetro & Temperatura & 18 & Física \\
\hline 1952 & Abr. & Eletricidade; Bússola; Balanças & Eletricidade; Posicionamento; Massa & 18 & Física \\
\hline 1952 & Maio & $\begin{array}{l}\text { Observar alavancas; Chuva; } \\
\text { Densidade; Calor }\end{array}$ & $\begin{array}{l}\text { Alavancas; Estados físicos da água; } \\
\text { Densidade da água; Sensação térmica }\end{array}$ & 16 & $\begin{array}{l}\text { Física e } \\
\text { Química }\end{array}$ \\
\hline 1952 & Ago. & $\begin{array}{l}\text { Como as plantas são úteis para o } \\
\text { homem?; Como os animais são } \\
\text { úteis para o homem? }\end{array}$ & Relações interespecíficas & 14 & Biologia \\
\hline 1953 & Mar. & $\begin{array}{l}\text { As raízes respiram; As folhas } \\
\text { transpiram; Como plantar? }\end{array}$ & Plantas & 15 & Biologia \\
\hline 1953 & Set. & Uma árvore; $\mathrm{O}$ pessegueiro & Plantas & 18 & Biologia \\
\hline 1954 & Mar. & $\begin{array}{c}\text { Germinação e crescimento } \\
\text { vegetal }\end{array}$ & Plantas & 22 & Biologia \\
\hline 1954 & Abr. & Tropismo & Plantas & 29 & Biologia \\
\hline 1954 & Maio & Mecânica & Forças e alavancas & 14 & Física \\
\hline 1954 & Jun. & $\begin{array}{c}\text { Propriedades gerais e estados } \\
\text { físicos }\end{array}$ & $\begin{array}{l}\text { Compressibilidade e elasticidade, } \\
\text { porosidade, pressão, equilíbrio de } \\
\text { líquido e gases }\end{array}$ & 29 & Física \\
\hline 1954 & Out. & Termologia & $\begin{array}{l}\text { Condutibilidade das substâncias, } \\
\text { dilatação linear }\end{array}$ & 25 & Química \\
\hline
\end{tabular}


RELACult - Revista Latino-Americana de Estudos em Cultura e Sociedade

Revista Latinoamericana de Estudios en Cultura y Sociedad | Latin American Journal of Studies in Culture and Society V. 05, ed. especial, abr., 2019, artigo n 1213| claec.org/relacult |e-ISSN: 2525-7870

\begin{tabular}{|c|c|c|c|c|c|}
\hline 1955 & Mar. & A água & Estados físicos da matéria & 20 & Química \\
\hline 1955 & Jun. & $\begin{array}{c}\text { Propriedades essenciais da } \\
\text { matéria }\end{array}$ & Estados físicos da matéria & 20 & Química \\
\hline 1955 & Ago. & Inércia & Leis de Newton & 17 & Física \\
\hline 1956 & Out. & Os quatro elementos dos adubos & Elementos químicos & 14 & Química \\
\hline 1957 & Mar. & O uso didático do aquário & $\begin{array}{l}\text { Locomoção e respiração dos peixes; } \\
\text { Locomoção dos girinos; Metamorfose }\end{array}$ & 36 & Biologia \\
\hline 1957 & Maio & O uso didático do aquário & Plantas & 16 & Biologia \\
\hline 1957 & Ago. & O uso didático do aquário & Invertebrados & 16 & Biologia \\
\hline 1957 & Set. & O uso didático do aquário & Invertebrados & 08 & Biologia \\
\hline 1957 & Out. & O museu inanimado & $\begin{array}{c}\text { Coleta, conservação e classificação de } \\
\text { insetos }\end{array}$ & 09 & Biologia \\
\hline 1957 & Nov. & O museu inanimado & $\begin{array}{c}\text { Conservação de animais e vegetais em } \\
\text { meio aquoso }\end{array}$ & 08 & Biologia \\
\hline 1958 & Mar. & Respiração & Sistema respiratório & 19 & Biologia \\
\hline 1958 & Set. & Experiência com sementes & Plantas & 12 & Biologia \\
\hline 1958 & Out. & Causa do dia e da noite & Astronomia & 15 & Física \\
\hline 1958 & Nov. & Peso do ar & Peso e massa & 10 & Física \\
\hline 1959 & Abr. & Evaporação & Estados físicos da matéria & 20 & Química \\
\hline 1959 & Jun. & Combustão; Ilusão de ótica & Combustão; Ótica & 19 & $\begin{array}{l}\text { Física e } \\
\text { Química }\end{array}$ \\
\hline 1959 & Ago. & Acústica & Ondas & 25 & Física \\
\hline 1959 & Set, & Germinação & Plantas & 33 & Biologia \\
\hline
\end{tabular}

Fonte: STOLL, et al. $(2018, \mathrm{~s} / \mathrm{p}$.

Acredita-se que a predominância em Biologia se deve ao fato da RE/RS ter como foco o ensino primário e o pré-primário. Levando em consideração o contexto histórico delimitado, a primeira Lei de Diretrizes e Bases da Educação foi promulgada em 1961. Antes disso, ministravam-se aulas de Ciências Naturais apenas nas duas últimas séries do antigo curso ginasial. O que indica que possivelmente as experimentações de Física e Química, bem como algumas de Biologia não eram destinadas às crianças. Neste sentido, este achado possibilita 
como perspectiva de pesquisa futura a busca e análise do Currículo Escolar de Ciências Naturais da década de 1950 e as relações com o atual currículo.

Destacam-se também as edições de 1957, que orientam professores a como desenvolver experimentações com o uso didático do aquário (março, maio, agosto e setembro) e a criar um museu inanimado (outubro e novembro), através da coleta e conservação de insetos, animais e vegetais. Nessas edições, que possuem uma temática específica, o responsável pela publicação foi o Prof. Newton Dias dos Santos e as informações contidas são excertos do livro "Práticas de Ciências: Guia do Ensino Elementar", da Editora Olímpia, publicado com a autorização do autor. A partir das edições de 1958 as propostas de experimentações tornam-se aleatórias novamente.

\section{Considerações Finais}

A partir deste levantamento inicial realizado nas edições da Revista do Ensino/RS, no período de 1951 a 1959, evidenciou-se que as propostas de experimentações contidas nas Seções "Observando" e "Observações e Experiências" assemelham-se às práticas experimentais utilizadas atualmente nas aulas de Ciências da Natureza. Ao classificar as experimentações e destacar os conteúdos que podem ser abordados, cria-se a possibilidade para um aprofundamento teórico-metodológico futuro, bem como, (re)aplicação dessas atividades em sala de aula.

Salienta-se que a RE/RS possui outras seções destinadas ao Ensino de Ciências, nas quais apresenta reportagens, jogos didáticos e práticas educativas sobre a área, constituindo-se como um novo campo de pesquisa a ser explorado.

\section{Referências}

BASTOS, M. H. C. As Revistas Pedagógicas e a Atualização do Professor: a Revista do Ensino no Rio Grande do Sul (1951 - 1992). In: BASTOS, M. H. C.; CATANI, D. B. Educação em revista - A imprensa periódica e a história da educação. 1 ed. São Paulo: Escrituras, 1997, p. 47-76.

A revista do Ensino do Rio Grande do Sul (1929-1942): o novo e o nacional em revista. Pelotas: Seiva, 2005. 192 p.

BICA, A. C. A Organização da Educação Pública Municipal no Governo de Carlos Cavalcanti Mangabeira (1925-1929) no município de Bagé/RS. 2013. 301 f. Tese (Doutorado em Educação) - Universidade do Vale do Rio dos Sinos, São Leopoldo. 2013. 
CARVALHO, A. M. P. de; VANNUCCHI, A. I. ; BARROS, M. A.; GONÇALVES, M. E. R.; REY, R. C. Ciências no Ensino Fundamental - O Conhecimento Físico. 1. ed. São Paulo: Editora Scipione, 1998. 200 p.

CUNHA, M. L. O. de. As práticas corporais e esportivas nas praças e parques públicos da cidade de Porto Alegre (1920-1940). 2009. 116 f. Dissertação (Mestrado em Ciências do Movimento Humano) - Universidade Federal do Rio Grande do Sul, Porto Alegre. 2009.

DUARTE, C; G. A - realidade - nas tramas discursivas da educação matemática escolar. 2009. 191 f. Tese (Doutorado em Educação) - Universidade do Vale do Rio dos Sinos, São Leopoldo. 2009.

FARIA, J. B. de. Os quadros parietais nas escolas do Sudeste brasileiro (1890-1970). 2017. 332 f. Tese (Doutorado em Educação: História, Política e Sociedade) - Pontifícia Universidade Católica de São Paulo, São Paulo. 2017.

GIL, A. C. Como elaborar projetos de pesquisas. 4. ed. São Paulo: Atlas, 2002. 175 p.

GIORDAN, M. O papel da experimentação no ensino de ciências. Revista Química Nova na Escola, n. 10, p. 43-49, nov., 1999. ISSN 2175-2599. Disponível em: http://qnesc.sbq.org.br/online/qnesc10/pesquisa.pdf. Acesso em: 14 jan. 2019

LIMA, L R. F. de C. Ensinando na cozinha! Investigando a prática pedagógica de professores e a experimentação nas séries iniciais. 2016. 132 f. Dissertação (Mestrado em Educação em Ciências) - Universidade Estadual de Santa Cruz, Santa Cruz. 2016.

MINAYO, M. C. de S. Pesquisa social: teoria, método e criatividade. In: DESLANDES, S. F.; NETO, O. C.; GOMES; R.; MINAYO, M. C. de S. 21 ed. Petrópolis: Vozes, 2002, p. 930 .

OLIVEIRA, J. R. S. Contribuições e abordagens das atividades experimentais no ensino de ciências: reunindo elementos para a prática docente. Acta Scientiae, Canoas, v. 12, n. 1, p. 139-153, jan./jun., 2010. ISSN 2317-8957. Disponível em: http://w3.ufsm.br/laequi/wpcontent/uploads/2015/03/contribui\%C3\%A7\%C3\%B5es-e-abordagens-de-atividadesexperimentais.pdf. Acesso em: 14 jan. 2019.

PEREIRA, L. H. F. Os discursos sobre a matemática publicados na revista do Ensino do Rio Grande do Sul (1951-1978). 2010. 315 f. Tese (Doutorado em Educação) - Pontifícia Universidade Católica do Rio Grande do Sul, Porto Alegre. 2010.

ROSITO, B. A. O ensino de ciências e a experimentação. In: MORAES, R. Construtivismo e ensino de ciências: reflexões epistemológicas e metodológicas. 3. ed. Porto Alegre: EDIPUCRS, 2008, p. 195-208.

SARDAGNA, H. V. Práticas normalizadoras na educação especial: um estudo a partir da rede municipal de ensino de Novo Hamburgo, RS (segunda metade do século XX início do 
século XXI). 2008. 315 f. Tese (Doutorado em Educação) - Universidade do Vale do Rio dos Sinos, São Leopoldo. 2008.

STOLL, V. G; BELES, N. S. de M.; BICA, A. C.; COUTINHO, C. O Ensino de Ciências na Revista do Ensino/RS: levantamento da Seção Observações e Experiências. In: SALÃO INTERNACIONAL DE ENSINO, PESQUISA E EXTENSÃO, 10, 2018, Santana do Livramento. Anais... Santana do Livramento, 2018. Disponível em: http://seer.unipampa.edu.br/index.php/siepe/article/view/34235/19669. Acesso em: 14 jan. 2019.

UNIPAMPA - Universidade Federal do Pampa - Repositório Digital Tatu. Disponível em: http://sistemas.bage.unipampa.edu.br/tatu/. Acesso em: 14 jan. 2019. 